\title{
Effectiveness of educational video in improving oral hygiene in preschool students
}

\author{
Rinintha Adistia ${ }^{1}$, Wade'ah Wafa ${ }^{2}$, Eriska Riyanti ${ }^{1}$, Arlette Suzy Puspa Pertiwi \\ Setiawan ${ }^{*}$ \\ 1Department of Pediatric Dentistry, Faculty of Dentistry Universitas Padjadjaran, Indonesia \\ ${ }^{2}$ Department of Paediatric Dentistry \& Orthodontics, Faculty of Dentistry University of Malaya, \\ Malaysia
}

\begin{abstract}
Introduction: In recent years, attention has been drawn toward assessing the effectiveness of an oral health education program, especially for preschool students, in order to improve oral hygiene status. There is a difference in the level of ability and learning methods based on age. For early childhood, they tend to imitate the things they see, both people and multimedia images. This research was aimed to analyse the effectiveness of educational video in improving oral hygiene level in preschool students. Methods: This type of research was quasi-experimental with one group pretest-posttest design. The sampling method was the total sampling and obtained 40 subjects. The research population was all students in Al Azhar Kindergarten aged 4-6 years and met the inclusion criteria. Greene and Vermilion Plaque Index was applied to measure the oral hygiene status of each subject before and after the intervention with educational video. The intervention consisted of four meetings; each meeting consisted of before and after plaque index measurement and also after watching the educational video. Data were analysed using t-paired statistical analysis. Results: The result showed a comparison of difference of the plaque index value change on the day I with day VII, which obtained the $t$-value $=3.365$, higher than the $\mathrm{t}$-table $=2.02$. Conclusion: Educational video is effective in improving oral hygiene in preschool students by reducing the plaque accumulation.
\end{abstract}

Keywords: Plaque, educational video, preschooler.

p-ISSN: 1979-0201; e-ISSN: 2549-6212; Available from: http://jurnal.unpad.ac.id/pid/article/view/19312 DOI: 10.24198/pid.vol32no2.19312

Submission: Nov 14, 2018; Accepted: Jul 20, 2020; Published online: Jul 31, 2020

"Corresponding author: Arlette Suzy Puspa Pertiwi Setiawan, Department of Pediatric Dentistry, Faculty of Dentistry Universitas Padjadjaran, Indonesia. Department of Pediatric Dentistry, Faculty of Dentistry Universitas Padjadjaran, Jalan Sekeloa Selatan I, Bandung, West Java, Indonesia, 40132. Phone: +6281395309191; Email: arlette.puspa@fkg.unpad.ac.id 


\section{INTRODUCTION}

Oral disease qualifies as a significant public health problem owing to their high prevalence and incidence in all regions of the world, including Indonesia. The results of the Indonesia Basic Health Research (Riskesdas) 2018 stated that $93 \%$ of early childhood within the age of preschoolers had cavities. ${ }^{1}$ Preschool children are at risk for dental disease. Various factors have been identified to affect children's oral disease including poor oral hygiene. ${ }^{2-4}$ Such variable should be consid-ered when developing oral health education programs targeting preschool children.

The irony of the problem is that most oral diseases are preventable, but unfortunately, adequate efforts are lacking in that direction. The model for risk factor approach in the promotion of oral health is the role of modifiable risk behaviours such as oral hygiene practice. Oral health education is an essential component of oral health promotion, which aims to pro-mote oral health primarily by providing information to improve good oral health behaviour. ${ }^{5,6}$ Dentist plays a vital role in the improvement of the public's oral health education. One of the main objectives of dental education is to motivate pa-tients/community to adopt good oral hygiene as a part of general oral health..$^{7-9}$ To maintain oral health, tooth brush-ing is found to be the most effective hygiene practice, thus must always be included in developing dental education materials. ${ }^{10,11}$

Toothbrushing, like all habits of hygiene, is acquired during the socialisation process of the child. When this habit is taught in early childhood, e.g. preschoolers, it is naturally ingrained in the daily routine of the child. Oral hy-giene instruction through educational lectures results in highly significant improvement in oral health. ${ }^{6,12}$ However, there is evidence that the development of grammatical understanding in very young children continues for several years. This could explain the difficulty found in the training and practice of oral hygiene techniques in preschool chil-dren using only verbal instruction. The use of audiovisual methods is suggested for these children. ${ }^{6,13}$ The purpose of this study was to analyse the effectiveness of educational video in improving oral hygiene level in preschool students.

\section{METHODS}

The study has been approved by the ethical committee and written informed consent has been given by parents or guardians prior to the study, allowing their children to participate. The study was a quasi-experimental with one group pretest-posttest design. The manipulation of an independent variable occurred without designation of a control group or randomisation of the participant. In pre-posttest studies, a measurement occurred before and after each participant was exposed to the intervention. Thus, each child served as his control for evaluation of the effect of educational video on their oral hygiene. The study was conducted in Al Azhar Kindergarten, from January to March 2013.

An alpha of 0.05 and power of 0.80 was assumed to calculate the sample size, in addition to a minimum difference of two adequate behaviours before and after the intervention, which results in a minimum sample of 32 subjects. The inclusion criteria of the study were as follows: students in Al-Azhar Kindergarten aged 4-6 years old; having complete free caries in their primary dentition, and with medium to high plaque index (as a measure for oral hygiene). All children who met the inclusion criteria were invited to participate, resulting in $\mathbf{4 0}$ children.

As for the study protocol beginning, all children included in the study had their plaque index examined with Green Vermillion Oral Hygiene Index. Two drops of disclosing solution was given on the base of the tongue. Then the subjects were instructed to close their mouth and perform the gargle movement so that the liquid can be evenly distributed by saliva to the entire surface of the tooth. After examining the plaque index, subjects were shown an educational video which contained how to brush their teeth (by Fones method) and how to maintain their oral health followed by toothbrushing practice super-vised by all researchers. Plaque index was examined at the end of the meeting, and subjects were given a one-week check-list to their parents to fill in after toothbrushing routine (after breakfast and before bedtime at night).

The intervention consisted of three meetings after the first meeting (day II, IV, 
and VII) aimed at educating children to adopt appropriate behaviours related to tooth brushing. The reason for choosing those examining days was according to the character of plaque, which is the young plaque at day II, the old plaque at day IV, and the change of nature at day VII. ${ }^{14}$ Subjects was asked not to perform toothbrushing in the morning of each meeting day. Each consecutive meeting consisted of plaque index examination, watch educational video, toothbrushing practice, and finalised by plaque index examination.

Categorical variables were presented according to absolute and relative frequencies, and descrip-tive statistics were used for numerical variables, including mean, standard deviation, minimum and maximum values. Because the data was normally distributed, a significance level of $5 \%$ was considered for analysis of association, and a paired t-test was also chosen for further analysis.

\section{RESULTS}

Changes in the index plaque before and after intervention can be seen in Figure 1. From the day I through the end of the observation period, there was found a downward trend, both seen in the series of plaque value before and after the intervention. Table 1 shows the mean value of plaque index change be-fore and after intervention using educational video. The lowest difference occurred at the last meeting, which was at day VII $(0.0519 \pm 0.0606)$.

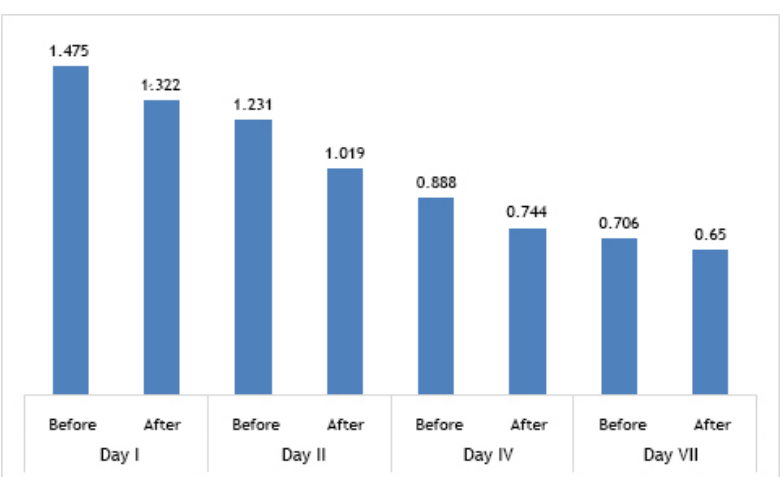

Figure 1. Overview of plaque index changes before and after educating using video on pre-school children of AlAzhar Kindergarten

Table 2 shows the result of statistical analysis on plaque index changes before and after intervention with educational video in every single meeting day using paired $t$-test at $a=5 \%$, yielding the highest t-count on day II (8.119). Every single meeting day shows a significant difference before and after intervention with educational video.

Table 1. Mean value of plaque index change before and after watching educational video in preschool children of Al-Azhar Kindergarten

\begin{tabular}{cccc}
\hline \multicolumn{4}{c}{ Plaque index changes } \\
\hline Day & Before & After & Difference \\
\hline 1 & $1.3112 \pm 1.6388$ & $1.1191 \pm 1.4434$ & $0.1921 \pm 0.1954$ \\
2 & $1.1015 \pm 1.3610$ & $0.9023 \pm 1.1352$ & $0.1992 \pm 0.2258$ \\
4 & $0.7789 \pm 0.9961$ & $0.6680 \pm 0.8195$ & $0.1109 \pm 0.1766$ \\
7 & $0.6255 \pm 0.7870$ & $0.5736 \pm 0.7264$ & $0.0519 \pm 0.0606$ \\
\hline
\end{tabular}

Table 2. Result of paired t-test

\begin{tabular}{cccl}
\hline & t count & T table & \\
\hline Day-1 & 3.115 & 2.02 & Significantly different \\
Day-2 & 8.119 & 2.02 & Significantly different \\
Day-4 & 3.908 & 2.02 & Significantly different \\
Day-7 & 3.365 & 2.02 & Significantly different \\
\hline
\end{tabular}

\section{DISCUSSION}

Based on the results, the average of the Green Vermillion plaque index during one week of study showed a decrease in plaque index average. This is due to the start of new behavior in children, a sequential process when one adopts a new behavior of awareness, interest, evaluation, trial, and adoption..$^{15}$ The subject of the study is in the awareness stage when given the material on oral and dental health education, continues in the interest stage when the video is played, then the subject is in the evaluation stage during the video play. Subjects then are in the trial phase when the toothbrush is together, and is in the adoption stage at the time of transfer from the trial phase to the one-week checklist stage at home. Subjects are given reinforcement through this process to adopt new behaviors, namely by practicing shared toothbrush material, checklists provided to the subject's parents, and repetition of counseling at the examination one week after counseling.

Checklist is a list of items, a person's behavior. The checklist given to the parents of the study subjects is a control given to the process of behavioral formation in early childhood. Parents are the first teachers for children and play an important role in stimulating the development and behavior of children. ${ }^{16}$ Child time is also spent more with parents. The knowledge and attention 
of parents is very important in underlying the formation of behaviors that support or do not support oral hygiene of children. ${ }^{17}$

The t-test performed showed significant differences between plaque index before counseling and shortly after counseling, while the most significant difference was obtained between the momentary plaque inductive index and the week after intervention. Educational video is an effort made to improve oral health in children. Health education with a psychological approach to preschoolers is necessary for the effectiveness of the delivery of the given message. The use of video media, allowing children to receive material about oral health. ${ }^{17}$ Audiovisual aids are valuable tools in education, more so, in the field of health education, as these have a long-lasting impression on the target population. ${ }^{5}$ This is evidenced by the decreasing plaque index.

The oral health educational film was found to be effective in increasing oral health-related knowledge of the subjects in the study conducted by Shah ${ }^{5}$ support the result of this study. The embedding of video bringing oral health awareness in subjects leading to a better attitude in tooth brushing which yields to reducing plaque index. ${ }^{5}$ Educational intervention, such in this study have experienced considerable advances involving strategies of psychological and behaviour change. ${ }^{18}$ This was seen as in the reducing of plaque index, thus leading to a better oral hygiene.

\section{CONCLUSION}

This present study throws light on the effectiveness of educational video as alternative media for dental health education. Educational video is effective in improving oral hygiene in preschool students by reducing the plaque accumulation.

\section{REFFERENCES}

1. National Institute of Health Research and Development (NIHRD). Indonesia Basic Health Research (RISKESDAS) 2017-2018. Jakarta: Ministry of Health of the Republic of Indonesia; 2018. pp. 1-100.

2. Shirzad M, Taghdisi MH, Dehdari T, Abolghasemi J. Oral health education program among preschool children: An application of health- promoting schools approach. Health Promot Perspect. 2016; 6(3): 164-70. DOI: $10.15171 /$ hpp.2016.26

3. Shaghaghian $\mathrm{S}$, Zeraatkar M. Factors affecting oral hygiene and tooth brushing in preschool children. J Dent Biomater. 2017; 4(2): 394402.

4. Mahat G, Bowen F. Parental knowledge about urban preschool children's oral health risk. Pediatr Nurs. 2017; 43(1): 30-4.

5. Shah N, Mathur VP, Kathuria V, Gupta T. Effectiveness of an educational video in improving oral health knowledge in a hospital setting. Indian J Dent. 2016; 7(2): 70-5. DOI: 10.4103/0975-962X.184646

6. Sigaud CHDS, Dos Santos BR, Costa $P$, Toriyama ATM. Promoting oral care in the preschool child: Effects of a playful learning intervention. Rev Bras Enferm. 2017; 70(3): 519-25. DOI: 10.1590/0034-7167-2016-0237

7. Rahman B, Al Kawas S. The relationship between dental health behavior, oral hygiene and gingival status of dental students in the United Arab Emirates. Eur J Dent. 2013; 7(1): 22-7.

8. Kumar H, Behura SS, Ramachandra $S$, Nishat R, Dash KC, Mohiddin G. Oral health knowledge, attitude, and practices among dental and medical students in Eastern India A comparative study. J Int Soc Prev Community Dent. 2017; 7(1): 58-63. DOI: 10.4103/jispcd. JISPCD_30_17

9. Haque $\mathrm{SE}$, Rahman $M$, Itsuko $\mathrm{K}$, Mutahara $M$, Kayako S, Tsutsumi A, et al. Effect of a schoolbased oral health education in preventing untreated dental caries and increasing knowledge, attitude, and practices among adolescents in Bangladesh. BMC Oral Health. 2016; 16: 44. DOI: 10.1186/s12903-016-0202-3 10. Koyuncuoğlu CZ, Kazak M, Pamuk F, Çifcibașı $E$. Oral hygiene habits and oral health status of female adolescents under state protection: A pilot study. J Istanb Univ Fac Dent. 2017; 51(1): 1-7. DOI: 10.17096/jiufd.65898

11. Melo P, Fine C, Malone S, Frencken JE, Horn V. The effectiveness of the Brush Day and Night programme in improving children's toothbrushing knowledge and behaviour. Int Dent J. 2018; 68(Suppl 1): 7-16. DOI: 10.1111/ idj. 12410 
12. de Jong-Lenters M, L'Hoir M, Polak E, Duijster D. Promoting parenting strategies to improve tooth brushing in children: Design of a nonrandomised cluster-controlled trial. BMC Oral Health. 2019; 19: 210. DOI: 10.1186/s12903019-0902-6

13. Setiawan ASPP, Elsari L, Agustiani H. Intervention program on dental fear in Sekeloa Kindergarten Bandung Indonesia. J Int Dent Med Res. 2018; 11(2): 602-6.

14. Chetruş V, Ion IR. Dental plaque-classification, formation, and identification. Int J Med Dent. 2013; 3(2): 139-43.

15. Conner M, Norman P. Health behaviour: Current issues and challenges. Psychol Health. 2017; 32(8): 895-906. DOI: 10.1080/08870446.2017.1336240

16. Denboba A, Hasan A, Wodon Q. Early Childhood Education and Development in Indonesia:
An Assessment of Policies Using SABER. Washington: World Bank Publications; 2015. p. 70

17. Lemos LVFM, Myaki SI, Walter LRDF, Zuanon ACC. Oral health promotion in early childhood: Age of joining preventive program and behavioral aspects. Einstein (Sao Paulo). 2014; 12(1): 6-10. DOI: 10.1590/S1679. 45082014A02895

18. Prentice AM, Ward KA, Goldberg GR, Jarjou LM, Moore SE, Fulford AJ, et al. Critical windows for nutritional interventions against stunting. Am J Clin Nutr. 2013;98(3):854-5. DOI: $10.3945 / a j c n .112 .052332$

19. Mohammadi TM, Hajizamani A, Bozorgmehr E. Improving oral health status of preschool children using motivational interviewing method. Dent Res J (Isfahan). 2015; 12(5): 476-81. DOI: $10.4103 / 1735-3327.166231$ 